\title{
Supporting Information \\ Performance of Inverted Quantum Dot Light-Emitting Diodes Enhanced by Using Phosphorescent Molecules as Exciton Harvesters
}

\author{
Guohong Liu ${ }^{1,2}$, Xiang Zhou ${ }^{1 *}$, Xiaowei Sun ${ }^{2}$, Shuming Chen ${ }^{2 *}$ \\ ${ }^{1}$ State Key Lab of Optoelectronic Materials and Technologies, School of Materials \\ Science and Engineering, Sun Yat-Sen University, Guangzhou, 510275, P. R. China \\ stszx@mail.sysu.edu.cn \\ ${ }^{2}$ Department of Electrical and Electronics Engineering, South University of Science \\ and Technology of China, Shenzhen, 518055, P. R. China \\ chen.sm@sustc.edu.cn
}



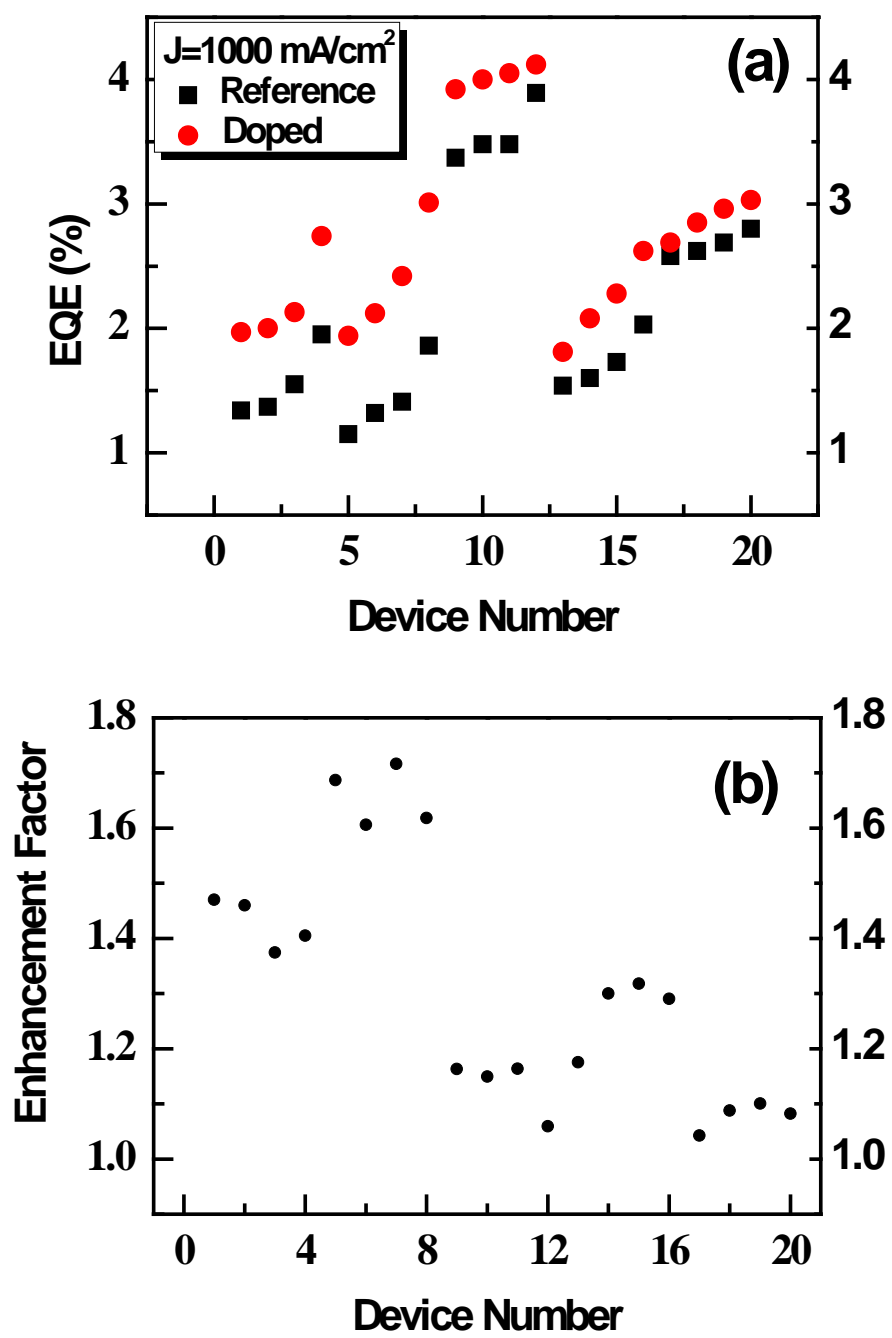

Figure S1. (a) EQE distribution of 20 groups of devices (normal device and device with 5 $\mathrm{nm}$ TCTA:FIrpic) and (b) corresponding EQE enhancement factor at the current density of $1000 \mathrm{~mA} / \mathrm{cm}^{2}$. 

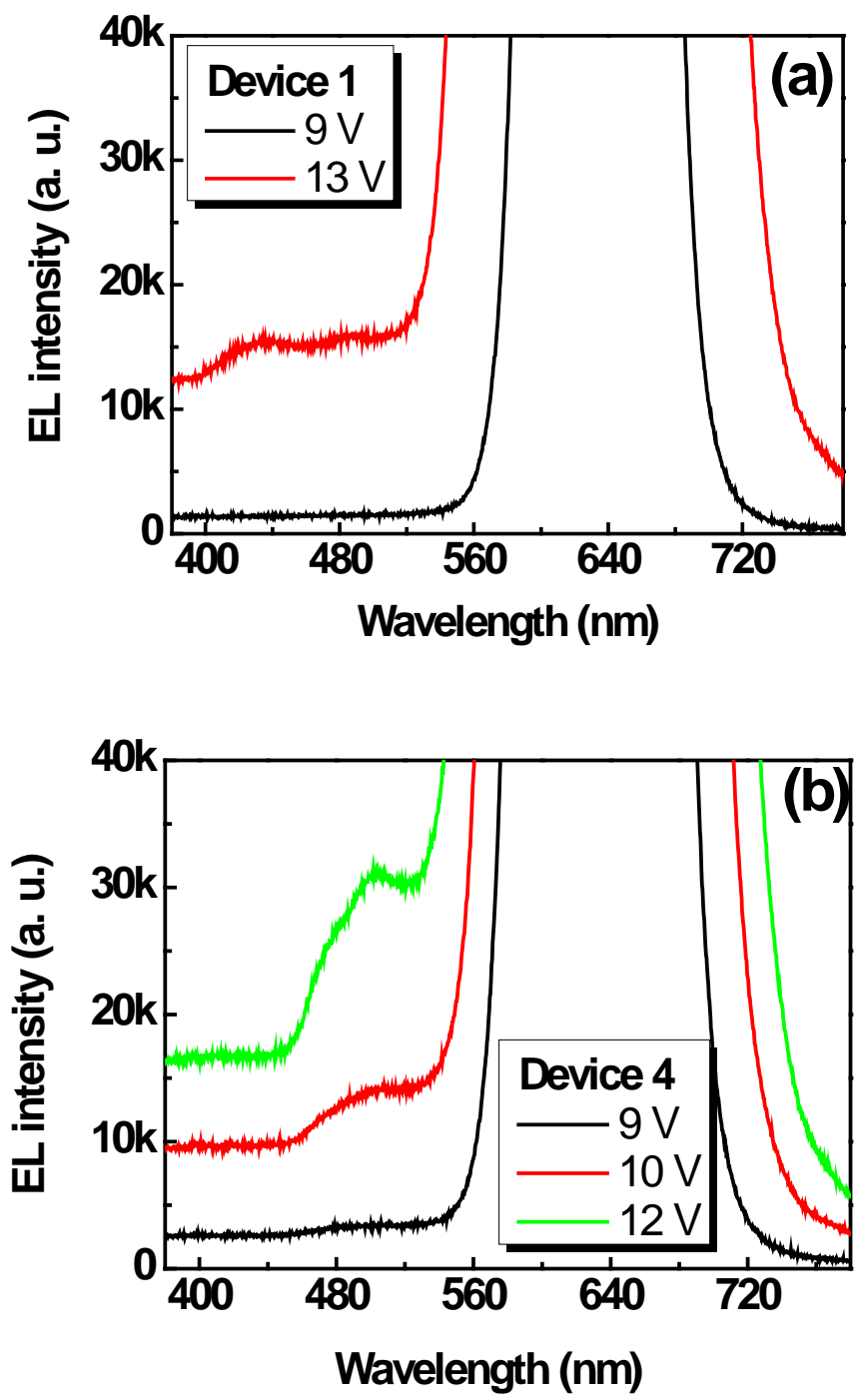

Figure S2. (a) EL spectra of the reference device 1 which showed NPB emission peaks at high voltages of $13 \mathrm{~V}$. (b) EL spectra of device 4 with $10 \mathrm{~nm}$ TCTA:Firpic at high voltages from $9 \mathrm{~V}$ to $12 \mathrm{~V}$. the spectral shape become more distinguishable at higher voltages such as $12 \mathrm{~V}$. 

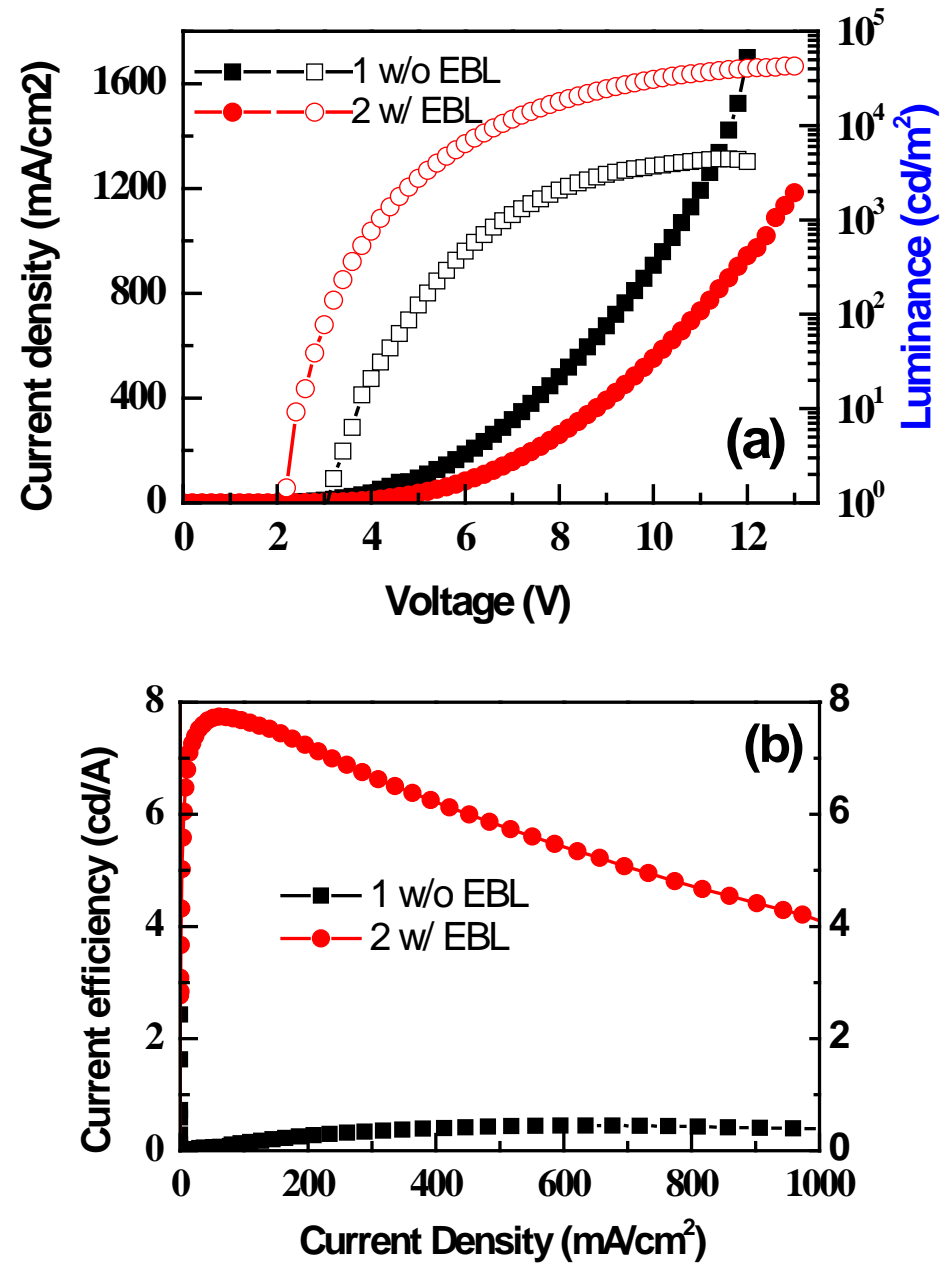

Figure S3. The (a)J-V-L and (b)current efficiency-J characteristics of the QD-LEDs with structure (device1:ITO/ZnO (55)/QD (26)/NPB 50/HATCN 20/Ag 80, and device 2:ITO/ZnO (55)/QD (26)/TCTA 30/NPB 20/HATCN 20/Ag 80)

It is apparent that, our QD-LED with CdSe/CdS/ZnS QDs, is extremely inefficient in the absence of the TCTA EBL, showing very large current density and rather weak emission as compared to the QD-LED with $30 \mathrm{~nm}$ TCTA EBL. The efficient electron blocking effect of TCTA is also investigated in reference (Electron Device Letters, IEEE 2015, 36(4):369-371) that we cited. Note that the efficiency of device 2 here is larger than that of device 4 in the manuscript because of replacing Al with highly reflective $\mathrm{Ag}$ as top electrode. 